\title{
Occipital nerve stimulation in a patient with an intractable chronic headache
} -A case report-

\author{
Jae Hyuck Shin, Yong Chul Kim, In Ki Jang, Jae-Hun Kim, Soo Young Park, and Sang Chul Lee \\ Department of Anesthesiology and Pain Medicine, Seoul National University College of Medicine, Seoul, Korea
}

Occipital nerve stimulation (ONS) is a form of peripheral nerve stimulation used to treat refractory headaches. The trial of ONS was carried with the midline incision C1-2 level, inserted electrical lead subcutaneously to oblique and cephalad direction followed by trajectory of blunt dissection. We used 8 pole electrical lead to cover lesser occipital nerve, greater occipital nerve, third occipital nerve and great auricular nerve. We anchored the lead at the midline insertion site after confirming the stimulation of the patient. And then we looped and tightened the lead loosely, connected the lead and the extension under right supraspinatus muscle region. After 1 week trial period, we performed the permanent implantation of occipital nerve stimulator. We inserted internal pulse generator under a pocket located at right infraclavicular region. The VAS score dropped from 8/10 to $1-2 / 10$. No serious complications were detected during 1 month follow-up. (Korean J Anesthesiol 2011; 60: 298-301)

Key Words: Headache, Occipital nerve stimulation, Occipital neuralgia, Peripheral nerve stimulation.

Neuromodulation is a growing area of chronic pain management, and anesthesiologists are sometimes called upon to support neuraxial or peripheral nerve stimulator insertion procedures. Occipital nerve stimulation (ONS) is a form of peripheral nerve stimulation that is used to treat refractory headache disorders [1]. ONS is a useful tool in the treatment of chronic severe headaches with at least level IV (limited) evidence based on multiple positive studies [2,3]. A growing body of literature describes the benefits of this technique for patients suffering from cluster headache [4], migraine [5], occipital neuralgia [6], postsurgical pain [7] and hemicranias continua [8].

A number of complications, including lead migration, infection, lack or loss of effect, localized pain and muscle spasms have been reported after ONS [9]. Among them, lead migration is one of the most serious and troublesome complications. Many authors have reported this complication with spinal cord stimulation [10,11].

The purpose of this report is to introduce new surgical techniques and to describe our first experience with permanent

Received: June 30, 2010. Revised: 1st, July 15, 2010; 2nd, September 2, 2010. Accepted: December 30, 2010.

Corresponding author: Yong Chul Kim, M.D., Department of Anesthesiology and Pain Medicine, Seoul National University College of Medicine, 28, Yeongeon-dong, Jongno-gu, Seoul 110-744, Korea. Tel: 82-2-2072-2467, Fax: 82-2-747-5639, E-mail: pain@snu.ac.kr The 50th scientific meeting of the Korean Pain Society, 2010, Daegu, Korea.

(c) This is an open-access article distributed under the terms of the Creative Commons Attribution Non-Commercial License (http:// creativecommons.org/licenses/by-nc/3.0/), which permits unrestricted non-commercial use, distribution, and reproduction in any medium, provided the original work is properly cited. 
ONS placement performed under minimal sedation. We will also review the medical literature regarding the techniques of ONS.

\section{Case Report}

A 71-year-old female patient presented with a 12-year history of occipital headache and posterior neck pain. Until her visit to our pain center, the patient had been receiving medications and various interventions, including supraorbital nerve block, C2 dorsal root ganglion (DRG) block, occipital nerve block and stellate ganglion block. The Visual Analogue Scale (VAS) score of her pain was $8 / 10$ and the pain nature was stabbing and intermittent. The pain continued for $3-4$ hours per episode. The pain site was mainly the right occipital area and was not associated with aura, dizziness and vertigo. Her medications included a tramadol hydrochloride $37.5 \mathrm{mg} /$ acetaminophen $325 \mathrm{mg}$ complex tabliet $0.5 \mathrm{~T}$ PO t.i.d., clonazepam $0.25 \mathrm{mg}$ PO t.i.d., tianeptin $12.5 \mathrm{mg}$ PO b.i.d. A bone scan showed no active bone or joint lesions.

Cervical spine X-ray findings showed mild cervical spondylosis, no significant foraminal narrowing and multiple calcified lymph nodes in the right neck. Because previous interventions had no effect or only short-term effects, we performed right C2 DRG pulsed radio frequency ( $\mathrm{pRF}$ ) intervention. Two weeks later, we carried out a right third occipital nerve (TON) block and the right $\mathrm{C} 4$ medial branch of a dorsal ramus block. The VAS score of the pain dropped to 4-5/10 after each procedure but the effects did not last long. Hence, we proposed an occipital nerve stimulation trial and she agreed.

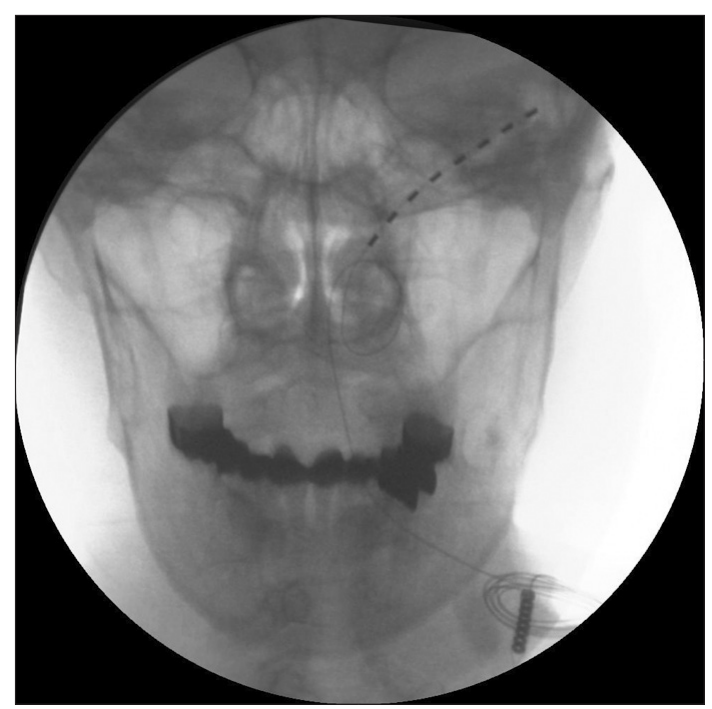

Fig. 1. This fluoroscopic image is an intraoperative fluoroscopic image after the occipital nerve stimulation trial.
After informed consent from the patient, the trial was carried out after hair removal with clippers and a razor in the occipital region. Sterile preparation of the surgical field was used, and antibiotic prophylaxis was given before the skin was punctured. The patient was placed in the prone position and given a local anesthesia plus minimal sedation. We dissected subcutaneously to oblique in the cephalad direction up to the retromastoid site at the C1-2 level using a hemostat and a Kelly hemostat. A lead with 8 electrical poles was then inserted through the dissection through a guide tube with a hole. Fluoroscopy was frequently used to identify anatomic landmarks (e.g., the $\mathrm{Cl}$ arch, mastoid process) and document the lead location (Fig. 1). The lead was powered with an external power source. The stimulation test was performed and the patient was satisfied with the stimulation program. The lead was fastened with an anchor at the midline incision site and was then looped and tightened loosely at the midline incision site (Fig. 2). We tunneled from the midline incision to the right supraspinatus muscle of the scapula region subcutaneously to connect the lead and the extension. In the pocket, the lead and the extension were looped and the extension, via another subcutaneous tunnel from the supraspinatus pocket to the right rhomboides muscle of the scapula region, was then fastened and taken out of the body. Finally, we sutured the midline incision and the pocket and applied antibiotic ointment and dressing. She was given a medical neck collar and admitted as an inpatient. During her inpatient stay, the patient expressed her satisfaction with nerve stimulation with 1.2 volts amplitude but at 1.3 volts, she felt a mild itching sensation at the occipital area. Other complications were not detected. Her VAS score became 2/10 during her

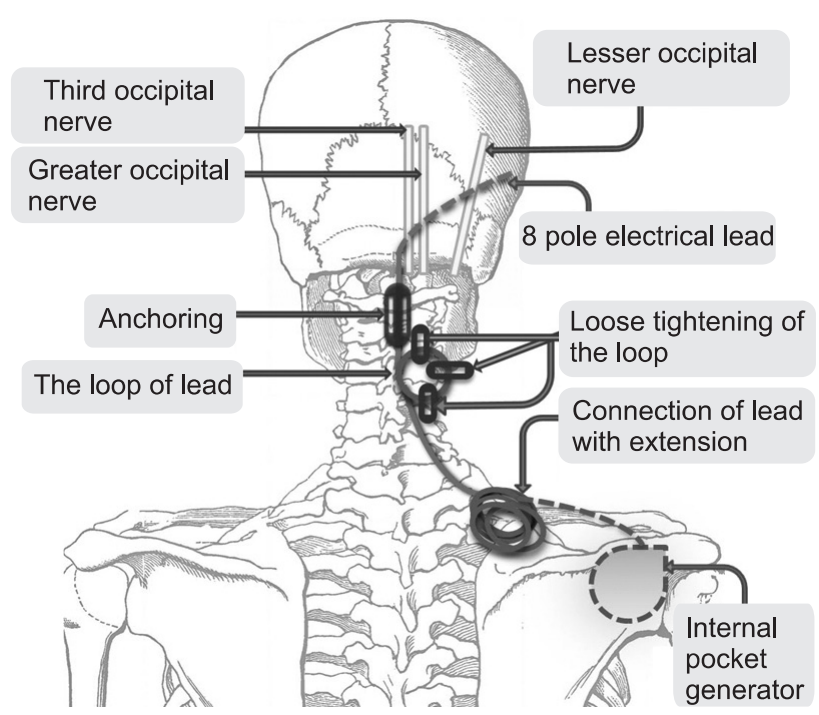

Fig. 2. A schematic drawing is about the occipital nerve stimulation procedure. 
admission. She was discharged 2 days later, and we, with her in agreement, decided to do a permanent implantation of an occipital nerve stimulator after a one-week trial period.

As in the trial, sterile preparation of the surgical field was used and antibiotic prophylaxis was given before the skin was punctured. The patient was placed in the lateral decubitus position instead of the prone position because we designed a pocket for an internal pulse generator (IPG) at the right infraclavicular region. She was given local anesthesia plus minimal sedation. After we reconfirmed that the lead position and the stimulation pattern were satisfactory, a silicone anchor was used with non-absorbable sutures to attach the lead to the fascia at the midline incision site. Finally, the lead was tunneled toward the IPG pocket. The IPG was placed in the right subcutaneous infraclavicular region. After ONS was assessed for impedance, the incisions were closed in the layers following hemostatic inspection and irrigation. We then applied antibiotic ointment and dressing. She was admitted to monitor for any complications. The day after the implantation, the patient was discharged with the caution of limiting her neck motion. Upon a one-week follow-up, her VAS score was low, at $1-2 / 10$, but she felt discomfort in her right infraclavicular pocket region. We removed the sutures 3 days later. Upon a one-month follow-up, the pain control continued to work, and she reported that the discomfort in the infraclavicular pocket region had decreased. The amount of her medication was also reduced.

\section{Discussion}

Occipital nerve stimulation may offer a good modality for refractory headache disorders. This report introduces new surgical techniques and describes our first experience with permanent ONS placement.

Two technique of the incision for permanent ONS implantation have been described in the literature, as far as the authors know [9]. The first technique is the midline approach and the second technique is the lateral approach. The first approach is carried out through a midline incision at the $\mathrm{Cl}$ level, where the lead is inserted with a lateral trajectory from that point. The second approach is made via an incision medial and inferior to the mastoid process at the $\mathrm{C} 1$ level [12]. The midline approach has several advantages compared to the lateral approach. First, one incision is sufficient to achieve stimulation of one or both greater occipital nerves. Moreover, this approach can be used to stimulate the lesser occipital nerves and great auricular nerves [13]. Second, lead migration may be less frequent because strain on the lead extension occurs only with flexion; minimal strain occurs with lateral flexion and rotation of the neck. Another advantage is compliance with the flexion of the neck provided by the loose lead extension loop positioned at the incision site
[13]. Third, because the location of the incision, at the C1 level, is under the hair, cosmetic problems arise less compared to the lateral approach. We choose the midline approach due to these advantages, particularly for the reduced possibility of lead migration.

The lateral approach is performed using an incision made medial and inferior to the mastoid process again at the $\mathrm{C} 1$ level. Trentman et al. suggested several advantages of the lateral approach [12], including the comfortableness of the supine position of the patient during the procedure and the ease of the surgical approach, which minimizes mechanical stress on the components.

Our oblique cephalad trajectory in midline approach, as far as the authors know, has not been reported. There is a greater possibility, the authors believe, for the electrical lead wire to kink or break through the horizontally lateral trajectory in the midline approach after the procedure because the angle between the direction of the midline incision and the horizontally lateral trajectory is sharp. On the other hand, an oblique cephalad trajectory has an arch but not an angle, lessening the potential for kinking or breaking of the lead wire after the procedure.

We performed blunt dissection of subcutaneous tissue using a hemostat and a Kelly hemostat instead of a Tuohy needle. Blunt dissection may reduce surgical bleeding and may allow the lead to be placed at an adequate depth.

An eight-pole electrical lead can stimulate the greater occipital nerve (GON), lesser occipital nerve (LON), third occipital nerve (TON) and even the great auricular nerve simultaneously. First, it is easy to change the stimulation site and patterns by modulating the stimulation program. It is also possible to stimulate newly involved nerves in addition to those already involved.

A number of complications have been reported after ONS. These can include lead migration, infection or sepsis, hematoma formation, insufficiency or loss of effect, muscle spasms and rapid battery depletion. Fortunately, we did not experience any serious complications, apart from a mild itching sensation of the occipital area. The itching sensation was reported to have disappeared seven days after implantation in a followup. Lead migration is the most serious complication of ONS, but the mechanism of ONS lead migration is not completely understood. Clearly, the highly mobile neck region places stress on system components, which is not encountered during spinal cord stimulation. Neck movement, flexion, and lateral rotation at the waist, and even arm movement can impact the leads and extensions [9]. There are no outcome studies to guide surgeons as to the best techniques to minimize lead migration. The use of silicone glue, although we did not use, may reduce failure of the anchor/lead interface [9]. Franzini introduced transfixation 
of the lead with a suture needle [14]. Medical neck collars for 10 days post implantation can be another option for preventing lead migration by minimizing neck movement [13]. To prevent lead migration, we initially anchored the lead tightly with a silicon anchor at the midline incision level. Second, we looped the lead and loosely tightened it at the midline incision site to avoid stretching stress on the lead caused by neck movement.

For successful trial and implantation, frequent checking of the ONS lead position using a $\mathrm{C}$ arm during the trial and implantation and careful bleeding control to prevent hematoma formation are necessary. For trial insertion of the leads, the procedure can be performed under conscious sedation, whereas general anesthesia can be used for permanent implantation.

This study described new ONS implantation techniques. Through these techniques, the probability that complications such as lead migration, bleeding and lead kinking will arise are lessened, and an adequate tunneling depth and coverage of the GON, LON, TON and great auricular nerves are secured. However, it is a matter of course that long-term follow-up and additional studies will provide better clarification of the best surgical techniques for this challenging form of peripheral nerve stimulation.

\section{References}

1. Bartsch T, Paemeleire K, Goadsby PJ. Neurostimulation approaches to primary headache disorders. Curr Opin Neurol 2009; 22: 262-8.

2. Jasper JF, Hayek SM. Implanted Occipital Nerve Stimulators. Pain Physician 2008; 11: 187-200.

3. Schwedt TJ, Dodick DW, Hentz J, Trentman TL, Zimmerman RS. Occipital nerve stimulation for chronic headache -long-term safety and efficacy. Cephalalgia 2007; 27: 153-7.

4. Burns B, Watkins L, Goadsby PJ. Treatment of medically intractable cluster headache by occipital nerve stimulation: long-term followup of eight patients. Lancet 2007; 369: 1099-106.

5. Rogers LL, Swidan S. Stimulation of the occipital nerve for the treatment of migraine: current state and future prospects. Acta Neurochir Suppl 2007; 97: 121-8

6. Johnstone CS, Sundaraj R. Occipital nerve stimulation for the treatment of occipital neuralgia - eight case studies. Neuromodulation 2006; 9: 41-7.

7. Ghaemi K, Capelle HH, Kinfe TM, Krauss JK. Occipital nerve stimulation for refractory occipital pain after occipitocervical fusion: expanding indications. Stereotact Funct Neurosurg 2008; 86: 391-3.

8. Schwedt TJ, Dodick DW, Trentman TL, Zimmerman RS. Occipital nerve stimulation for chronic cluster headache and hemicranias continua: pain relief and persistence of autonomic features. Cephalalgia 2006; 26: 1025-7.

9. Trentman TL, Zimmerman RS. Occipital nerve stimulation: technical and surgical aspects of implantation. Headache 2008; 48: 319-27.

10. Andersen C. Complications in spinal cord stimulation for treatment of angina pectoris. Differences in unipolar and multipolar percutaneous inserted electrodes. Acta Cardiol 1997; 52: 325-33.

11. Burchiel KJ, Anderson VC, Brown FD, Fessler RG, Friedman WA, Pelofsky S, et al. Prospective, multicenter study of spinal cord stimulation for relief of chronic back and extremity pain. Spine 1996; 21: 2786-94.

12. Trentman TL, Slavin KV, Freeman JA, Zimmerman RS. Occipital nerve stimulator placement via a retromastoid to infraclavicular approach: a technical report. Stereotact Funct Neurosurg 2010; 88: 121-5.

13. Kapural L, Mekhail N, Hayek SM, Stanton-Hicks M, Malak O. Occipital Nerve Electrical Stimulation via the Midline Approach and Subcutaneous Surgical Leads for Treatment of Severe Occipital Neuralgia: a Pilot Study. Anesth Analg 2005; 101: 171-4.

14. Franzini A, Messina G, Leone M, Broggi G. Occipital nerve stimulation (ONS). Surgical technique and prevention of late electrode migration. Acta Neurochir (Wien) 2009; 151: 861-5. 